\title{
Disclosure, Stress, and Support During Gender Transition on Facebook
}

\author{
Oliver L. Haimson, Jed R. Brubaker, Lynn Dombrowski, Gillian R. Hayes \\ Department of Informatics \\ University of California, Irvine \\ Irvine, CA, USA \\ \{ohaimson, jed.brubaker, ldombrow, hayesg\}@uci.edu
}

\begin{abstract}
Social computing technologies, such as social networking sites (SNSs), often privilege people who fit within expected, static categories. Thus, users embarking on major identity changes, such as gender transition, often encounter stress when using SNSs to interact with their online social networks. To address this problem and reflect on the design of SNSs and other social computing systems, we present the results of a comprehensive online survey of transgender and gender non-conforming SNS users. Our findings indicate that although Facebook can be a stressful place for gender transition due to difficulties of transition disclosure, support from one's Facebook network can help to mitigate some of this stress. We examine Facebook both as a site of stress and as a site of support. Better understanding the relationships between stress, disclosure, and support on SNSs for these particular users can inform technology design that will benefit people who struggle with navigating a wide range of major identity changes online.
\end{abstract}

\section{Author Keywords}

Social networking sites; identity transitions; life transitions; transgender; LGBTQ; online identity; stress; support.

\section{ACM Classification Keywords}

H.5.3. Information interfaces and presentation (e.g., HCI):

Group and Organization Interfaces: Collaborative

computing, Computer-supported cooperative work, Web-

based interaction.

\section{INTRODUCTION}

Many people struggle with online self-presentation during periods of life transition. Announcing and representing changes on social networking sites (SNS) can cause stress and uncertainty. The pervasive nature of social media adds additional challenges and opportunities to a wide range of identity transitions, particularly those that involve potential

Permission to make digital or hard copies of all or part of this work for personal or classroom use is granted without fee provided that copies are not made or distributed for profit or commercial advantage and that copies bear this notice and the full citation on the first page. Copyrights for components of this work owned by others than ACM must be honored. Abstracting with credit is permitted. To copy otherwise, or republish, to post on servers or to redistribute to lists, requires prior specific permission and/or a fee. Request permissions from Permissions@acm.org.

CSCW '15, March 14-18 2015, Vancouver, BC, Canada. Copyright is held by the owner/author(s). Publication rights licensed to ACM. ACM 978-1-

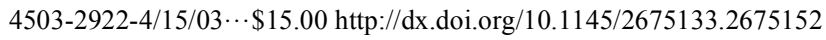

disapproval or estrangement from others in one's network (e.g., changes in relationship status, job termination, coming out as gay or lesbian, child birth, gender transition). The complexities that arise during identity transitions on SNSs warrant specific analysis of issues including disclosure concerns and the role of one's online social network in transition support.

Taking gender transition as a case study of identity transition more broadly, what particular SNS practices add to or diminish stress as individuals change gender on SNSs? Gender transitions have already been shown to be stressful offline [29]. In this work, we demonstrate that they are also stressful online. However, simply knowing that something is stressful is not enough. We must understand how and why these transitions are stressful to effectively design technology and SNSs that counter stress while leveraging one's online social network for transition support. Designing such systems with considerations for life transition support can reduce stress for transgender people and others during periods of identity transition.

In what follows, we describe ways in which gender transition on Facebook can be uniquely stressful, beyond the stress experienced from gender transition in general. We describe what kinds of Facebook account management activities are related to self-reported online transition stress. In particular, transition disclosure and concerns around how others will respond to this disclosure are major stressors. However, we also find that transition-related support from one's Facebook network may reduce this stress. Our analyses investigate Facebook both as a site of stress and as a site of support during gender transition.

In this work, we determine that navigating the parts of Facebook's user interface and infrastructure that relate to disclosure predict additional stress for this group of users, while support from one's Facebook network predict reduced stress. Additionally, Facebook's new gender options, a "fix" for some of the classification problems that the system has with transgender and gender nonconforming users, have not actually reduced transitionrelated stress for this group of users, likely due to problems of disclosure. These results give insight into the ways that sociotechnical systems can improve disclosure options and use social network support to reduce stress for those changing identities online. 
CSCW has a rich history of scholarship critiquing the uneven politics of classification and categorization (e.g., [5, 42]). Classification of gender and its relation to selfpresentation is a secondary theme of this paper and an important area for continued research. However, our findings show that addressing categorization issues alone is not enough. Disclosure of personal information about identity transitions on Facebook is a more fundamental concern for participants in this work. Therefore, in this paper, we focus primarily on five research questions related to differential identity disclosure, as outlined here:

Stress. How stressful is gender transition on Facebook, and how does this compare with stress experienced during gender transition in other settings?

Account management strategies and disclosure. In what account management activities do people engage during gender transition on Facebook, and how are these activities related to disclosure and associated with stress?

Network transition support. During gender transition, how supportive are peoples' Facebook networks, how does this compare with support received from other networks, and how is this support associated with stress experienced?

Custom gender options and disclosure. How do Facebook's new custom gender options relate to gender transitionrelated disclosure and stress?

Demographics. Are demographic variables such as race, gender, or socioeconomic status associated with higher levels of stress during gender transition on Facebook?

The remainder of this paper is organized as follows. First, we provide some background on transgender issues and identity transitions. Next, we discuss related research, followed by a description of our methods. We then present empirical findings based on analysis of data from our online survey. We close with a discussion of design implications and a summary of our findings.

\section{BACKGROUND}

This section provides background by first defining transgender, then situating it among other life transitions and identity changes. We then discuss the recent history of gender representation on Facebook.

\section{Gender Transition as Identity and Life Transition}

Transgender is an umbrella term that can broadly be defined as including "those who transition from one gender to another... and those who may not, including genderqueer people, cross-dressers, the androgynous, and those whose gender non-conformity is part of their identity" [27:12]. Importantly, a person's relationship to their gender is what matters here, not necessarily physical characteristics or changes. Following [29], we use "trans" for the remainder of this paper to refer to the broad transgender population, except when designating between different facets of the transgender community.

Little is known about how trans people navigate gender transition online. Studying this community of SNS users and their information practices as an extreme case of identity transition can lead to better understandings of the sources of stress during life transitions more broadly, particularly those that involve substantial changes to identity. This work also provides insight into identity transition-related constraints within SNSs and their corresponding information infrastructures and user interfaces. These insights inform our knowledge of how stress and constraints encountered on SNSs more generally affect those embarking on major identity changes.

Although many major life changes cause people to change their identities substantially, gender transition is unique because numerous complex identity changes are coupled with risks of discrimination, harassment, and rejection [22, 27, 29]. Gender transition often leads to severe marginalization as well as rejection and disapproval from friends, family members, and networks at work and school $[22,29]$. All of these factors contribute to stress for trans people [29], making transition especially difficult. At the same time, gender transition is one of the only life changes so drastic that a person often becomes literally unrecognizable to their friends, family, and online social networks. For example, during transition, a person's SNS profile will often change in three substantial ways: name, gender, and physical appearance in profile photo. These changes can occur months or even years apart from each other. If such changes are made without announcement, or if the announcement of gender change is not displayed or noticed by some members of the trans person's online social network, a person who was once a "friend" now appears as a stranger. Thus, gender transition requires many complicated decisions around disclosure. Facing discrimination, violence, and rejection, while at the same time managing complex disclosure decisions, may make a trans person feel uncomfortable and unfamiliar within their online social network, thus causing stress, frustration, and disengagement with the SNS.

Because few other life transitions encompass such potentially drastic, numerous, and complex identity changes, gender transition allows insight into SNS practices in a way that other major life changes do not. Previous research found that students changing gender allowed university information systems administrators to uncover structural holes in databases [12, 30]. Likewise, studying the experiences of trans SNS users gives us a unique view into the potentially problematic nature of SNSs during identity transitions, which we will examine in the remainder of this paper.

\section{Gender Representation on Facebook}

Recently, the needs of trans people have received popular attention with Facebook's introduction of custom gender 


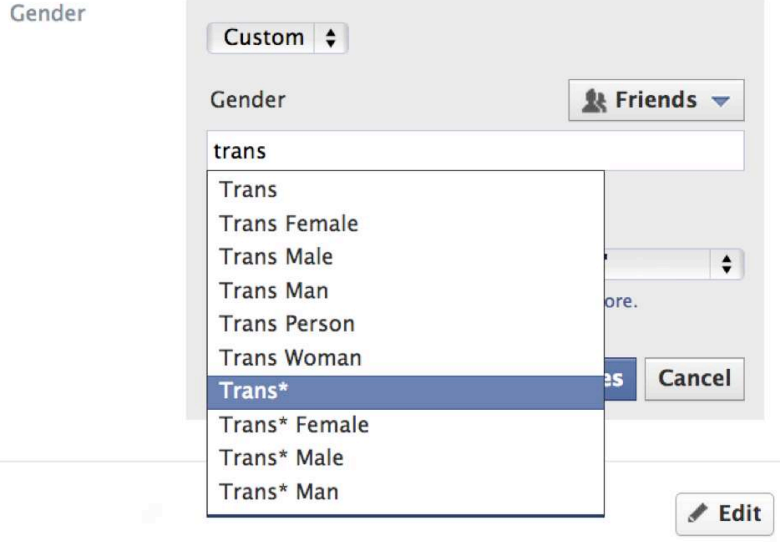

Figure 1. Custom gender option selection on Facebook [screenshot taken May 26, 2014].

options. On February 13, 2014, Facebook activated its new "Custom" gender options. Instead of only two gender options, users could now choose from 58 terms to describe their gender. Additionally, users could choose from three rather than two pronoun options. However, behind the user interface, Facebook's database continues to store gender in simplified ways, effectively categorizing many of its 56 custom gender options into more prominent gender codes [4]. This simplified classification system results in instances in which Facebook's underlying categories, which can be problematic for the trans user, bleed through into the various places in the system in which gendered language is used (e.g., the pronouns attached to an individual's activity) [4]. Inputting a custom gender is also problematic for some trans users, because the system imposes constraints on gender representation. While the interface includes what appears to be a freeform text box, the user is actually limited to a pre-set list of options that become visible as the user attempts to type in their preferred gender term (see Figure 1).

The recent implementation of additional gender options on Facebook is a laudable step in supporting the trans community. However, features that allow trans people to control the display of their gender identities cannot mitigate the much broader set of stressors of which self-presentation is only a part. In fact, in some cases, as we describe in this paper, the availability of additional options may create additional stress. In this work, we describe a broad range of account management activities, including those related to disclosure and representation, in terms of their potential to both create and diminish stress related to gender transition on Facebook.

\section{RELATED WORK}

Researching the trans population provides a unique contribution to existing $\mathrm{CSCW}$ literature on major life changes. With the exception of [4] and [31], no work has focused specifically on trans people's use of SNSs, and no empirical research has been conducted in this area. Thus, we present a review of related work about stress, support, and disclosure on SNSs during life transitions more broadly.

Social computing systems, such as SNSs, allow users unique ways to express identity online during major life changes and can be major sources of social support [11] and helpful information for adapting to changes [41]. Studies show that online social networks and support groups benefit a wide range of transitional communities, such as new parents [20], those who have recently lost a job [11], adults transitioning to old age [37], and students entering college $[19,39,41,43]$. In the absence of socialization that allows people to gain the skills necessary to smoothly navigate life transitions [25], some of these skills are learned via SNS networks. Networks on SNSs are an important source of support for people facing major life changes.

However, SNSs can also be potentially harmful spaces during life transitions. The public, open nature of SNSs can place users at risk of harassment, which can complicate and even impede life transitions online [16, 36]. Communication with one's Facebook network can cause added stress during life transitions when friends offer unhelpful advice, and passive consumption of news feed content can decrease social support [11]. Additionally, SNSs open up a whole new set of "digital possessions," such as photographs, messages, and even SNS profiles themselves, many of which must be sorted through and changed during a life transition [38]. By further examining the potentially negative aspects of using SNSs during life transitions, we seek to understand how to best support transition processes online, including ways to preserve privacy and allow for optimal network support.

In particular, SNSs complicate life transitions due to complexities around disclosure of transition-related information and self-presentation online. People carefully curate their self-presentation depending on their intended audience [26]. Impression management [26] has always been an important topic in SNS research [7], but is particularly relevant when considering complex and multiple identities that emerge during major life changes. On SNSs, people commonly present different information depending on the audience [6, 15, 23, 34, 35]. Maintaining multiple SNS profiles is a common practice, but is a burden and comes with the risk of unintended "leakage" between accounts [15]. Particularly for marginalized groups, having incompatible faceted identities may cause people to worry more about posting on Facebook, thus leading them to use email, a private platform, more often [23]. However, even with email, people maintain multiple accounts for use in different settings [28]. People use both mental strategies and account management behaviors on Facebook to "divide the platform into separate spaces" in order to manage disclosures among different groups of friends [32:288]. Customizing privacy settings, for instance creating lists of friends, is a common account management technique for disclosure, and has been shown to increase the amount of 
content shared on Facebook [40]. However, many Facebook users misinterpret privacy settings [1], which can lead to information being shared with unintended audiences $[34,47]$. Although users employ many strategies to manage disclosure on SNSs during life transitions, in this work we show that some of these behaviors are associated with increased transition-related stress.

Stress associated with life transitions [25, 41] can carry over to, or even be exacerbated by, SNSs. Our contribution to $\mathrm{CSCW}$ lies in understanding which Facebook account management behaviors relate to increased stress during transition, and how support from one's network may diminish this stress. In turn, by documenting the conditions under which the stress and support occurs, this work sheds light on how SNS designers can help reduce transitionrelated stress and promote network support.

\section{METHODS}

We developed an online survey aimed at trans people who had experienced gender transition on SNSs. Due to the nature of the research questions, the survey excluded people who had transitioned prior to establishing a profile on a SNS, because they would not have experienced gender transition on a SNS. Participants were recruited and asked to complete the survey via several methods. The authors shared recruitment materials on their social media profiles and encouraged our networks to share the link. Recruitment materials were emailed to several email lists targeted at the transgender and LGBTQ communities, and shared on LGBTQ and transgender-focused groups or forums on Facebook and other SNSs. We also distributed recruitment materials to our contacts at several health centers specializing in trans healthcare. Participation was incentivized by entering survey participants into a drawing for a $\$ 500$ Visa gift card. The survey was active April $15-$ May 31, 2014

Most of the questions provided Likert-style response options $(N=51)$ or yes/no with other possible response options $(N=16)$, while others provided lists of possible SNS practices with an "Other (please specify)" option that included a write-in answer box $(N=8)$. Other optional questions were more qualitative in nature and provided free-form write-in answer fields $(N=15)$. Many questions were shown to some participants but hidden to others depending on answers to previous questions. All survey questions relevant to the results presented in this paper are included in an Appendix at the end of the paper.

While our survey included questions about a variety of SNS platforms, the data and findings we report in this paper are specific to questions about Facebook (Q3-18). Because Facebook is the dominant SNS in the U.S., used by $71 \%$ of online adults [18] and by $98.90 \%$ of participants in our study, we focused on Facebook specifically to collect a large sample of data about one platform. This allowed us to analyze participants' gender transition practices and challenges on Facebook in more depth. Facebook is also particularly interesting, because the platform recently implemented custom gender options, which we analyze in relation to self-reported transition stress.

The first part of the survey (Q3-14) focused on Facebook use. Those who had previously maintained a Facebook profile but did not currently use Facebook were asked to reflect on "the time when you were last active on Facebook." Questions about Facebook touched on common practices regarding online transition account management activities in general (Q7) and management of one's "friends" list (Q8). Participants were then asked about how stressful it was to transition on Facebook (Q9a) and how many people in their Facebook network they perceived were supportive of their transition (Q10). These questions were followed by open-ended questions (Q11-14) such as "What parts of transitioning on Facebook were especially difficult? Why were they difficult?" and "What do you think would improve the online gender transition experience on Facebook?"

Next, respondents were asked about their thoughts and practices regarding Facebook's new custom gender options (Q15-18). Those who were not aware of the new custom gender options skipped this section of the survey. Questions then turned to stress experienced in particular offline settings (Q19-20) and self-perceived transition support received offline from friends, family, and co-workers (Q2124). Finally, demographic information was collected from participants (Q25-31).

Because a validated scale does not exist for measuring stress or support during gender transition, or even for measuring such variables during a previous time period, our stress and support variables instead measure self-perceived stress and support experienced during transition. We evaluated several validated stress and social support scales for possible use (e.g., $[13,33])$, but none were appropriate for evaluating stress and support experienced around a particular past event. Additionally, we were concerned that adding multi-question scales to an already lengthy survey would lower survey completion rates. However, our survey instruments exhibit face validity. To test the face validity of our stress variable, two of the authors qualitatively coded levels of stress represented in 35 randomly-selected participants' responses to open-ended questions. The average of the two coders' measures of qualitative stress was highly correlated with participants' Likert scale response to $\mathrm{Q} 9 \mathrm{a}, r(33)=.63, p<.001$, indicating face validity of Q9a as a measure of stress. Inter-rater reliability between the two coders was high at $r(33)=.86, p<.001$. Face validity was also demonstrated by positive feedback from a pilot group of survey participants.

Each section of the survey included optional questions for any additional information that participants wished to share (e.g., "Is there anything else you would like to tell us about your transition experience on Facebook?"). Answers to these and the aforementioned open-ended questions allowed 
us to qualitatively support our quantitative results using open coding of free-form response data. On average, for each of the open-ended questions about gender transition on Facebook, 55.81\% of participants opted to spend the extra time to include a write-in response.

\section{Data}

Our sample included 371 participants who completed the online survey. 615 participants started the survey but some did not finish, either being disqualified due to not meeting the study's criteria, or opting not to finish for other reasons. The first author manually cleaned the data, removing responses from participants whose answers appeared illogical or insincere, or who completed the survey despite not qualifying. Data from 62 participants was not used because they either were not a Facebook user or had not transitioned on Facebook. Our final model included data from 242 participants after removing participants with missing data for any of the relevant variables. All of these participants had experienced a gender transition of some sort on Facebook, whether from male to female, female to male, or from a binary gender to a non-binary gender such as genderqueer, agender, or gender non-conforming.

Participants were allowed to choose multiple gender and race/ethnicity options (for instance, a person may identify both as transfemale and as genderqueer, or as both white/Caucasian and Hispanic/Latino). As such, percentages add up to greater than $100 \%$. When asked their current gender, $46.30 \%$ of participants identified as female/ transfemale/transwoman/MTF, $39.18 \%$ as male/transmale/ transman/FTM, and $25.48 \%$ as genderqueer, agender, gender non-conforming, or a different gender. Our sample was not racially diverse: $90.68 \%$ were white/Caucasian, $5.21 \%$ were Asian/Pacific Islander, 5.48\% were Hispanic/Latino, $3.01 \%$ were American Indian/Native American, and 1.92\% were black/African-American. The lack of racial diversity may be a result of our networks used to recruit participants, and is a limitation of this research. The mean age was $28.93(S D=8.84$, range: $18-66)$.

Participants reported a median of approximately 201-500 Facebook friends and a median daily Facebook usage of approximately 1-2 hours. On average, participants began their transition on Facebook 2.41 years prior to taking the survey $(S D=2.07$, range: $0-10)$ and began their transition overall 3.97 years prior to taking the survey $(S D=4.60$, range: 0-45).

We cannot claim that our sample is representative of the trans population overall. It is not currently possible to obtain a random sample of trans people [27], however, the most comprehensive and representative survey of trans people to date is Grant et al.'s National Transgender Discrimination Survey [27]. Our sample is comparable to Grant et al.'s [27] in terms of gender identity when categorizing participants as male, female, or other, $X^{2}(2, N$ $=371)=3.39, p=.18$. However, significant racial differences exist between our sample and Grant et al.'s
[26], $X^{2}(4, N=371)=20.41, p<.001$. Specifically, our sample includes significantly fewer blacks/AfricanAmericans (standardized residual $=-3.32$ ), fewer American Indians/Native Americans (standardized residual $=-2.24$ ), and more Whites/Caucasians (standardized residual $=2.71$ ) than Grant et al.'s [27], a limitation of our study.

\section{Data Analysis}

Quantitative data were analyzed using descriptive statistics and statistical modeling to explore relationships between stress, support, and account management activities, the variables of interest in this work. Stress around transition disclosure also emerged as a common theme in qualitative open-coding of short-answer responses by two of the authors.

To understand which disclosure-related activities (see Table 1) most contributed to and reduced stress, we used regression models to explore the relationships between account management activities and self-reported transition stress. We first ran 11 separate regressions, one for each account management activity in Table 1, with self-reported stress as the outcome variable and the account management activity as an independent variable along with control variables. Then, only those account management activities that predicted increased stress of transition on Facebook were used as independent variables in a final standardized linear regression model (see Table 2). This model included self-reported stress as the outcome variable, and selfreported transition practices on Facebook and level of support from one's Facebook network as independent variables. Control variables included gender, age, years since transition, race/ethnicity, socioeconomic status indicators, amount of time spent on Facebook daily, and number of Facebook friends.

\section{Limitations}

Because our analysis is based on survey data, we present correlational results without causal claims. Our model includes control variables to eliminate potential confounds. This allows us to argue that real relationships exist between disclosure-related Facebook behavior, self-reported support and self-reported stress. Additionally, as described in the methods section, using non-validated survey instruments to measure stress and support is a potential limitation of this work. However, the face validity of our survey questions gives us confidence that our survey captured participants' self-perceived levels of stress and support during gender transition. Self-reported data is also a limitation because participants transitioned on average several years prior to taking the survey, leading to potential difficulties in recalling transition experiences. Next, as noted in the Data section, our sample may not be representative of the overall trans population, thus limiting the generalizability of our results. A final limitation is that peoples' lived experiences are more complex than can be captured by the online/offline binary that some of our survey questions assume. 


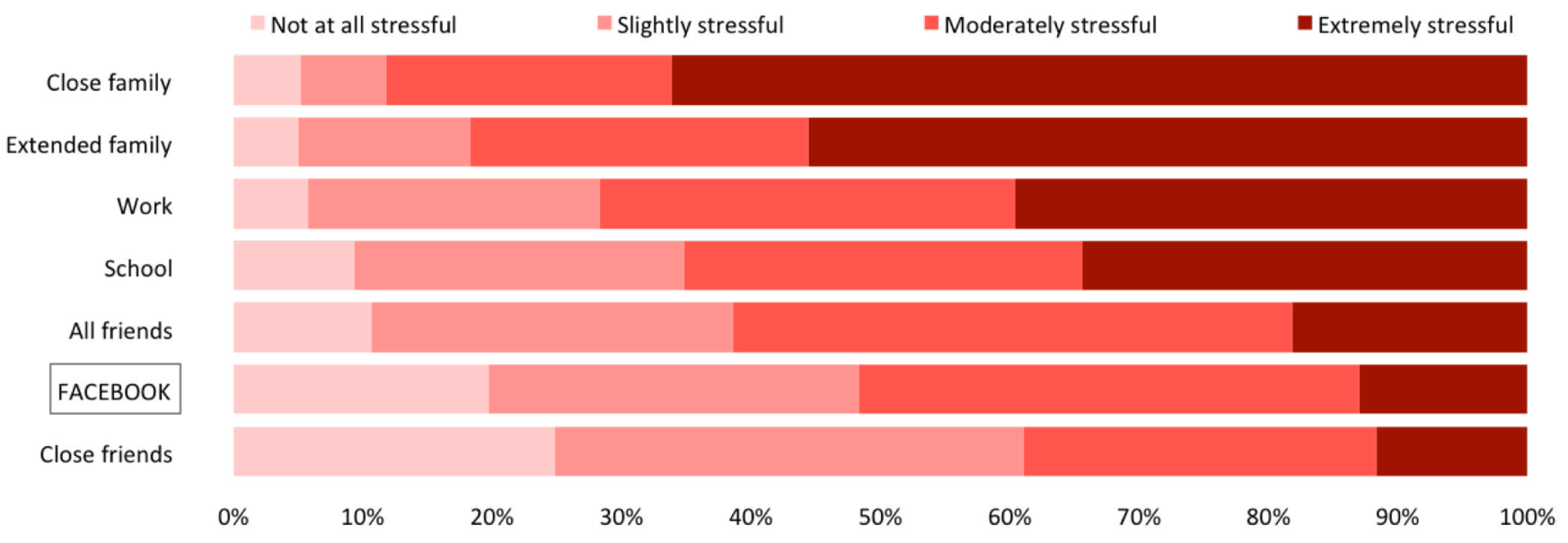

Figure 2. How stressful was it to transition in each of these environments? (Ordered from most stressful to least stressful)

\section{RESULTS}

The results of our work offer insight into the self-reported stress experienced by trans Facebook users and provide a statistical model examining relationships between stress and transition-related Facebook account management activities. These results also illuminate participants' perceived transition-related support among different networks, and how this relates to transition stress on Facebook in general and in light of Facebook's custom gender options. We also analyze demographic variables and transition-related stress.

\section{Stress}

How stressful is gender transition on Facebook?

During gender transition, trans people experience substantial stress while using Facebook. When asked how stressful it was to transition on Facebook (Q9a), 51.70\% of participants responded that it was "extremely stressful" or "moderately stressful", while only $19.73 \%$ described it as "not at all stressful" (see Figure 2).

How does this compare with stress experienced during gender transition in other settings?

Interactions on Facebook (Q9a) were reported to be significantly less stressful $(M=2.45, S D=.95)$ than interactions in most non-SNS transition settings (see Figure $2)$, such as among close family (Q19c) $(M=3.49, S D=$ $.85), t(282)=-16.95, p<.001$; among extended family (Q19d) $(M=3.34, S D=.89), t(261)=-13.10, p<.001$; at work $(\mathrm{Q} 19 \mathrm{e})(M=3.04, S D=.92), t(234)=-7.93, p<.001$; at school (Q19f) $(M=2.85, S D=1.00), t(178)=-5.00, p<$ .001 ; and among all friends (Q19b) $(M=2.69, S D=.89)$, $t(290)=-4.21, p<.01$. However, transitioning on Facebook was significantly more stressful than transitioning among close friends (Q19a) $(M=2.24, S D=.95), t(292)=3.38, p$ $<.01$. Although other transition settings cause people to experience more stress during gender transition, Facebook is unique because one's Facebook network often includes people from all of the categories considered most stressful close family, extended family, co-workers, classmates, and friends. During gender transition, an individual may want to present their identity differently to different social groups, thus complicating disclosure of transition-related information.

\section{Account Management Strategies and Disclosure}

In what account management activities do people engage during gender transition on Facebook, and how are these activities related to disclosure?

Participants engaged in many account management activities aimed at controlling disclosure, such as creating "lists" to share content with only certain friends, unfriending, switching to a new profile/account, and/or maintaining multiple profiles/accounts (see Table 1). Lists allow profile owners to disclose certain information only to particular groups of people. For example, separate lists can be maintained for family members, co-workers, and close friends. When posting a status update, the privacy setting can be toggled to restrict the audience of that post to only people appearing on particular lists. The ability to use lists to control disclosure may make transition on Facebook less

\begin{tabular}{lr}
\hline Activity & $\begin{array}{c}\text { Percentage who } \\
\text { engaged in activity }\end{array}$ \\
\hline Announced transition & $48.77 \%$ \\
Changed the gender listed on profile & $68.49 \%$ \\
Changed the name displayed on profile & $59.73 \%$ \\
Changed my profile picture & $62.74 \%$ \\
Deleted profile/account & $10.96 \%$ \\
Created new profile & $18.90 \%$ \\
Maintained multiple accounts & $14.79 \%$ \\
Unfriended or blocked people who may not be & $41.64 \%$ \\
supportive & $20.00 \%$ \\
Unfriended or blocked people I did not want to know & \\
about transition & $30.96 \%$ \\
Made some posts visible only to some people in & \\
network & $19.18 \%$ \\
Created lists or groups to make posts visible to only \\
certain groups of people
\end{tabular}

Table 1. Disclosure-related activities on Facebook. 
stressful than in other settings (see Figure 2). For example, monitoring what information a person discloses to their transphobic uncle on Facebook is much easier than doing so at a family gathering. However, the act of monitoring the audience of transition-related content and regularly scrutinizing friends lists may in fact contribute to some of the stress experienced on Facebook during transition.

How are these activities associated with stress?

Several important findings highlight the stressful nature of disclosure during transition. Two Facebook account management practices emerged as significant positive predictors of stress in our regression model (see Table 2):

- $\quad$ unfriending people whom a participant did not want to know about their transition (engaging in this practice predicted a 0.30 standard deviation increase in stress)

- creating lists or groups to make posts visible to only certain groups of people (engaging in this practice predicted a 0.40 standard deviation increase in stress)

Both of these practices directly relate to disclosure on Facebook. These associations between disclosure practices and stress highlight how complex management practices may actually increase stress, as we argue here. They may also, however, indicate that people experiencing more stress are more likely to engage in complex management practices in attempts to reduce stress. Many participants expressed concern with certain portions of their Facebook networks

\begin{tabular}{lcc}
\hline & \multicolumn{2}{c}{$\begin{array}{c}\text { Self-Reported Stress of } \\
\text { Transition on Facebook }\end{array}$} \\
\hline Variable & Coefficient & Standard error \\
\hline Unfriended people I didn't want to & $\mathbf{0 . 3 0 *}$ & $\mathbf{( 0 . 1 4 )}$ \\
know about my transition & & \\
$\begin{array}{l}\text { Created lists or groups to make posts } \\
\text { visible to only certain groups of people }\end{array}$ & $\mathbf{0 . 4 0 * *}$ & $\mathbf{( 0 . 1 5 )}$ \\
Perceived support from Facebook & $\mathbf{- 0 . 2 1 * * *}$ & $\mathbf{( 0 . 0 6 )}$ \\
network & & \\
Female & -0.04 & $(0.15)$ \\
Non-binary gender & -0.18 & $(0.19)$ \\
Age & -0.16 & $(0.09)$ \\
Years since transition & $-0.18 *$ & $(0.08)$ \\
American Indian/Native American & -0.05 & $(0.31)$ \\
Asian/Pacific Islander & -0.03 & $(0.27)$ \\
Black/African American & 0.15 & $(0.43)$ \\
Hispanic/Latino & 0.01 & $(0.24)$ \\
Highest degree & 0.14 & $(0.07)$ \\
Income & 0.10 & $(0.07)$ \\
Time spent on Facebook & -0.04 & $(0.06)$ \\
Size of Facebook network & 0.05 & $(0.07)$ \\
Intercept & -0.15 & $(0.11)$ \\
Adjusted $R^{2}=0.14, N=242$ & & \\
\hline$* p<.05 ; * * p<.01 ; * * * 0.001$ & & \\
\hline & & \\
\hline
\end{tabular}

Table 2. Results of standardized linear regression model examining factors associated with self-reported stress of transition on Facebook. finding out about their transition:

"[The] hardest part was making sure that what I decided to share ... remained only viewable to that group." (P268)

People can control the audience of their online transition to some degree through the various technological features supplied by Facebook. However, these tools do not account for people acting outside the system, such as by taking screenshots of Facebook content and sharing with people who are not friends with the profile owner, or other nonFacebook activities. Additionally, participants struggled to use or trust the audience control features provided:

"Because of Facebook's privacy settings, it's basically impossible to transition incrementally. (I imagine it's *technically* possible - but I don't know anyone who's actually understood the FB privacy setup well enough to pull it off.) So I had to be thoroughly closeted on FB until I was ready to post a completely public announcement." (P131)

"Because Facebook doesn't have an easy way of systematically sharing to/excluding circles of friends (like managing circles on $G^{+}$[Google+]), coming out or being myself with people on Facebook especially in the early stages of transition is really hard, or not worth the effort." (P143)

Understandably, the creation of a second account was a common pattern for "preventing 'leakage' between online personae" (P276). Although such practices did not significantly affect stress in regression models, almost $19 \%$ of participants reported creating a new Facebook account during their gender transition, and almost $15 \%$ maintained multiple Facebook accounts, perhaps due to the complexities of disclosure activities.

Engaging in account management practices allows trans Facebook users control over their transition disclosure, but in our model these practices also predicted increased stress. Severing ties to parts of one's network and keeping secrets from others is clearly stressful, and the social and public nature of Facebook requires trans users to actively manage friends lists throughout their transition. Additionally, the difficulty of navigating some account management activities may add to the stress of online transition.

\section{Network Transition Support}

During gender transition, how supportive are peoples' Facebook networks?

Facebook networks can also help reduce a trans person's stress during transition. Participants' networks on Facebook were generally perceived to be supportive (Q10), with $73.36 \%$ of participants reporting that they perceived that all, almost all, or most of the people in their Facebook network were supportive of their transition (see Figure 3). Only $11.84 \%$ perceived that few, almost none, or none of the people in their Facebook network were supportive. 


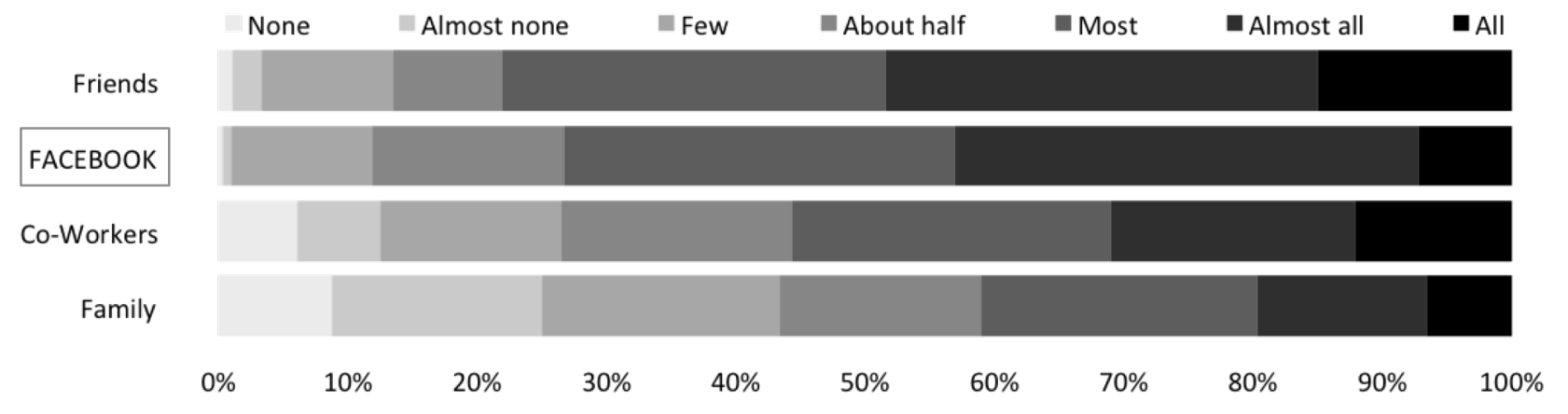

Figure 3. Roughly how many people in this network do you perceive were supportive of your transition online? (Ordered from most supportive to least supportive)

How does support on Facebook compare with support received from other networks during gender transition?

Compared to other networks (see Figure 3), participants' Facebook networks (Q10) were marginally less supportive $(M=5.11, S D=1.16)$ than their friends in offline settings (Q21) $(M=5.24, S D=1.32), U=50821, p=0.05$; but significantly more supportive than their co-workers (Q23) $(M=4.54, S D=1.66), U=50607.5, p<.001$; or family members (Q22) $(M=3.90, S D=1.72), U=78327, p<.001$ in offline settings.

How is transition-related support from one's Facebook network associated with stress during gender transition on Facebook?

The more supportive a respondent perceived their Facebook network to be of their transition, the less stressful they reported transition being on Facebook. From our model in Table 2, one standard deviation increase of support predicted a 0.21 standard deviation decrease in stress. Support from offline networks, such as family and coworkers, did not have a significant relationship with stress on Facebook, while one's offline friends network has a significant association with stress only before controlling for support from one's Facebook network.

A participant with a particularly supportive Facebook network described the ways that some friends expressed support in ways that may not have been possible offline:

"I came out in a letter I wrote to my friends list. All the people that commented on it had nothing but support to show me, and even some people sent me messages telling me that they could not publicly support me, but were happily supporting me behind closed doors." (P287)

Facebook can also provide access to new networks that have the unique ability to offer support for online transition:

\section{"The social circles of supporters and fellow trans people I've made on Facebook have provided substantial and vital support during my coming-out process." (P92)}

Because those with more supportive Facebook networks experienced less stress, we can see the potential for
Facebook network support to mitigate the stressful nature of managing transition-related information disclosure.

\section{Custom Gender Options and Disclosure}

How do Facebook's new custom gender options relate to gender transition-related disclosure and stress?

Facebook has recently made efforts to support trans people on its site by increasing options available for representing gender identity [21]. Notably, the new custom gender options, as described in the Background section above, provide numerous options for gender self-presentation. In its initial announcement about the new gender options, Facebook's Diversity team stated that its new custom gender options were introduced to help users to "feel comfortable being your true, authentic self" [21]. Among LGBT organizations [24] and in our survey responses, people praised Facebook for the usefulness of the new gender options. However, reported use of custom gender option(s) (Q15) has no statistical association with stress experienced by survey respondents during transition on Facebook. Those who used the custom gender options $(M=$ $2.50, S D=.97)$ and those who did not $(M=2.42, S D=.92)$ experienced similar levels of stress, $t(269)=-.65, p=.52$.

Using Facebook's custom gender options is a complex process of disclosure. Although the options are appealing in many ways, this complexity may in and of itself contribute to increased stress on Facebook. In particular, although being able to more accurately represent their gender on Facebook may reduce stress for some, this is counterbalanced by the stress of disclosing one's transgender or non-binary gender to one's social network.

Changing one's gender option to the opposite gender or to a non-binary identity is akin to disclosing one's transgender or non-binary status to one's entire network, or selected parts of one's network (by allowing only certain lists of people to see one's gender option). In our statistical model, we found that disclosure activities such as unfriending people and creating lists to control the audience for one's Facebook content are related to increased stress. Thus, it follows that trans people would be hesitant about or unwilling to change their gender option on Facebook. Changing one's gender option involves considerable 
contemplation about the audience to which one will disclose their gender change, as well as masterful navigation of Facebook's privacy settings. For instance, one participant stated:

"It actually worries me, to be honest. I think that
outing yourself as clearly transgender/gender non
confirming/etc - and having it visible by
EVERYONE - is dangerous. There are people out
there that target trans/gn-c [gender non-
conforming] people, and having that publicly listed
on your profile can set you apart as a target."
(P295)

Facebook privacy settings do allow individuals to control what audiences are able to see gender choices, but regardless, people often do not have a good understanding of who can view their profile content. Our qualitative data show that the challenges of maintaining privacy settings and understanding one's disclosure that has been noted elsewhere $[1,46]$ are particularly poignant in the case of gender transition.

Although most respondents appreciated Facebook's custom gender options $(80.00 \%$ said that they agreed or strongly agreed that these options improve the online gender transition experience in Q17a), only 39.18\% actually reported using the new gender options (Q15) at the time of data collection (roughly two months after the custom gender options were implemented). Custom gender options are used significantly more often by those trans people who identify outside of the male/female binary, such as genderqueer and agender people $(M=.70, S D=.46)$, than those trans people identifying with more binary genders $(M$ $=.29, S D=.45), t(363)=-7.54, p<.001$, and can indeed be a powerful way for some users to express their gender identity online:

"They're great for people who identify as
genderqueer, agender, bigender or one of the other
identities that falls outside the male/female binary."
(P310)
"I'm so happy that there are more gender options, it
was hard just seeing male. I feel that two-
spirit/trans/genderqueer represent me most
accurately." (P239)

Calling gender options "custom" suggests that individuals have the ability to tailor their disclosure based on the gender terms that they choose. However, at the time of this writing, gender options on Facebook are in fact not fully customizable. As noted in the Background section, Facebook gives the illusion of freeform text entry, but only allows users to select from a pre-set list (see Figure 1). When asked if they tried to enter a custom gender option that was not recognized by Facebook (Q16), 29.37\% of those using custom gender options reported that they had. Many participants expressed desires for truly customizable gender:
"I know that data collection is a primary focus of facebook. But damn it, custom gender should mean custom. A person should be able to gender themselves however they wish to, instead of choosing from a collection of preset options." (P11)

"The thing I've heard everyone lament (and I agree), is that there is no write-in option." (P137)

Such concerns are examples of problems that occur due to rigidity of the custom gender options and Facebook's underlying classification system. Since custom gender options map to much broader categories in Facebook's database [4], perhaps allowing users to truly customize the terms used to describe their gender could improve disclosure experiences while adding little overhead.

The combination of disclosure-related stress and the constraints that still exist around Facebook's custom gender options highlights the fact that simply giving people more gender options is not enough. To avoid privileging static identities, design of SNSs like Facebook should specifically address support for major identity changes.

\section{Demographics}

Are demographic variables such as race, gender, or socioeconomic status associated with higher levels of stress during gender transition on Facebook?

Gender transition on Facebook is stressful regardless of demographic factors. Demographic variables such as gender (i.e., sub-categories within the trans population) (Q25-26), race/ethnicity (Q27), and socioeconomic status (Q30-31) had no significant association with stress of gender transition online, although the non-randomness of our sample may have influenced these results. We found no significant difference between stress experienced by transgender men, transgender women, and those of nonbinary genders such as genderqueer and agender. Similarly, race/ethnicity did not significantly affect online transition stress. Socioeconomic status was measured in two ways: income and highest degree obtained, both of which had no significant association with transition stress on Facebook. Because gender transition on Facebook is equally stressful on average for those of different genders, races/ethnicities, and socioeconomic statuses, this points to a widespread need for technology to support transition for a broad array of the trans population.

\section{Summary of Results}

In summary, our results demonstrate that although Facebook can be a stressful place for gender transition due to difficulties of transition disclosure, support from one's Facebook network can help to mitigate some of this stress. Taken together, these results highlight the duality of Facebook as both a site of stress and a site of support. Given this, SNSs like Facebook as well as other platforms could be designed to reduce some of the existing disclosure-related stressors and simultaneously build on the positive nature of support during gender transition. 


\section{DISCUSSION}

During identity transitions on SNSs, many users are challenged with how and when to disclose content related to these changing identities. Identity is both multifaceted and fluid $[6,15,26,35]$, and our results indicate that this is particularly true during major life transitions. The crude support currently offered for complex, changing identities confirms the ways that SNSs often privilege normative selfpresentations $[35,45]$ and deem non-static concepts of self as problematic. In response to the ways that Facebook privileges those with static identities, trans users and others facing identity transitions adopt multiple accounts or actively manage disclosure practices as a way of negotiating technical constraints and social demands. However, our results indicate that such account management activities lead to stress.

The expectation of static identities on SNSs presumes that individuals come to SNSs to represent an already established identity, rather than to discover, invent, and reinvent themselves. However, in contrast to the authenticity that is now expected [35], online spaces were historically lauded for allowing people a space to explore and experiment with identities [10, 17, 44]. On SNSs, people are "not simply representing but constructing themselves" [45:426], a process that is highly influenced by SNS site design and policy that privileges certain identities while problematizing others [45].

Although we argue that the association between disclosure activities and stress highlights the difficulties and complexities of account management activities on Facebook, it is also useful to briefly explore the possibility of causality in the opposite direction. Perhaps increased stress of gender transition on Facebook causes people to use disclosure-related account management practices. Our qualitative data leads us to believe that stress is increased by navigation of complex account management activities, and not the other way around. However, further research is needed to fully understand the relationships between account management activities and stress, and in particular, whether those who use disclosure features in an attempt to reduce stress actually experience a reduction in stress after using these features.

Difficulties around identity transitions on Facebook extend beyond the user interface and into the database and system infrastructure. As we have demonstrated, Facebook's account management features for selective disclosure of transition-related content can be confusing and stressful. Similarly, Facebook's accommodation of non-binary genders via a list of pre-set gender options constrains gender presentation, causing frustration for some. Both point to larger issues about how Facebook operationalizes "identity" as a set of static attributes with distinct values [9].

Identity transitions are not as simple as pressing a button or flipping a switch - a person is not one day male, the next female. SNSs' emphasis on discrete states, and frequent inability to design for users that move between static categories, is consistent with emerging critiques in a growing body of work on use and non-use [2, 3, 8]. Existing systems often assume a binary - a person either uses a technology or doesn't [2], is married or isn't, is male or is female - when use practices and users themselves are instead much more complex The experience of using SNSs during identity transitions, gender transition on Facebook in particular, can in part be described as a kind of torque, the "twisting" that results from a mismatch between a person's identity and a classification system [5]. However, as shown by the lack of impact Facebook's new custom gender options had on stress, the design challenge is greater than just creating more categories. There remains a need for design that addresses torque and improves user experience for trans people and others who, during identity transitions, do not fit neatly into or move effortlessly between existing categories.

Many opportunities for enhanced system and SNS design arise regarding collaborative and social ways to support gender transitions on SNSs. Drawing from our finding that those with Facebook networks more supportive of their transition experience less stress during transition on Facebook, CSCW systems can help to encourage collaborative ways to support online transition. For instance, a system could enable members of a trans person's online social network to help their friend by providing support throughout the transition process in specific ways. Trusted friends could help by monitoring and/or removing digital artifacts that are non-representative and potentially harmful in light of a gender change (e.g., a photograph of a person prior to transition). Systems could include features to facilitate digital artifact management as collaborative work in support of life transitions. SNSs could also incorporate new ways for users to communicate support and empathy to those in their network who are facing a life transition. For instance, a SNS could allow people to publicly denote themselves as "trans friendly," thus alleviating from trans users some of the burden of identifying which friends to disclose transition-related information. Each of these SNS design enhancements, described here with respect to gender transition, could easily be modified to address other major identity changes.

Additionally, while we found a strong association between support from one's Facebook network and decreased stress around transition, not all Facebook users have a supportive network in place. Innovative methods could offer support resources to those who lack a social network of support. For instance, machine learning techniques could be employed to analyze profile text and network activity to determine users who may be facing transitions without network support. Such users could be targeted with transition-related and stress-reduction resources, and/or given supportive volunteers as "suggested friends." Additionally, a system could provide resources (such as relevant support groups, 
healthcare providers, and legal consultants) that may be helpful during transition. However, due to the sensitive nature of identity transitions and particularly gender transitions, great care should be taken to preserve user privacy and maintain ethical practices when employing such methods.

Finally, because users experience considerable stress around disclosure of gender change and gender identity, designers must consider the granularity [5] of gender options provided. As an example, Facebook's massive list of gender options, many of which are redundant, incorporates far too much granularity. A more appropriate approach would involve less granularity but more control over disclosure. For example, a simple but elegant solution would lump gender options into three categories - male, female, and custom/undisclosed - and would allow a freeform write-in box for the third option, as suggested for use in a healthcare context [14]. Such a design would also allow users to control which people or lists of people in their network can see the new gender option, and would display the user's gender as "undisclosed" to others in their network. This design would empower users to choose their own terms to represent their identities, as well as their own level of disclosure. For example, a trans person may simultaneously feel strongly about not identifying within the male/female binary and about not disclosing their trans identity to some in their online social network. A less granular infrastructure with more user control would allow such a person to identify outside of the binary without necessarily disclosing their trans status.

\section{CONCLUSION}

We present results from a comprehensive online survey targeted at trans SNS users examining stress, disclosure, and support throughout the gender transition process online. The results of this study demonstrate that specific Facebook account management activities impact stress during gender transition both positively and negatively. In particular, we argue that although practices around disclosure on Facebook are particularly stressful, online network support can mitigate some of this stress. Thus, technology and SNSs can be designed with a focus on disclosure and support in order to reduce the stress that accompanies online transition.

This work contributes to research on disclosure and social computing within the CSCW literature. In particular, lessons learned around disclosure, stress, and support during gender transition are likely to apply to those experiencing other "face threat" identity changes. Like those embarking on gender transition, these people likely also experience stress when interacting with user interfaces like Facebook's to limit the audience of certain content regarding their identity change. CSCW systems can help to leverage online social network support to reduce the stress around transition disclosure.

\section{ACKNOWLEDGEMENTS}

We thank our participants for sharing their experiences with us. Thank you to Karen Cheng, Rena Bivens, Anne Bowser, Ellie Harmon, members of STAR and LUCI, and our anonymous reviewers for their generous help and support with this paper. This work was funded in part by a UC Irvine Eugene Cota-Robles Fellowship.

\section{REFERENCES}

1. Acquisti, A. and Gross, R. Imagined communities: Awareness, information sharing, and privacy on the Facebook. In Privacy Enhancing Technologies. Springer, 2006, 36-58.

2. Baumer, E.P.S., Adams, P., Khovanskaya, V.D., et al. Limiting, leaving, and (re)lapsing: An exploration of Facebook non-use practices and experiences. Proceedings of CHI (2013).

3. Baumer, E.P.S., Ames, M.G., Brubaker, J.R., Burrell, J., and Dourish, P. Refusing, limiting, departing: Why we should study technology non-use. Proceedings of CHI Extended Abstracts (2014).

4. Bivens, R. The Gender Binary Will Not Be Deprogrammed: Ten Years of Coding Gender on Facebook. Social Science Research Network, 2014.

5. Bowker, G.C. and Star, S.L. Sorting Things Out: Classification and Its Consequences. MIT Press, 1999.

6. boyd, d.m. Friendster and publicly articulated social networking. Proceedings of CHI (2004).

7. boyd, d.m. and Ellison, N.B. Social network sites: Definition, history, and scholarship. $J$ of ComputerMediated Communication 13, 1 (2007), 210-230.

8. Brubaker, J.R., Ananny, M., and Crawford, K. Departing glances: A sociotechnical account of 'leaving' Grindr. New Media \& Society, (2014).

9. Brubaker, J.R. and Hayes, G.R. Select * from user: Infrastructure and socio-technical representation. Proceedings of CSCW (2011).

10. Bruckman, A.S. Gender swapping on the Internet. Proceedings of INET (1993).

11. Burke, M. and Kraut, R. Using Facebook after losing a job: Differential benefits of strong and weak ties. Proceedings of CSCW (2013).

12. Clarkson, N. "A Letter From Your Surgeon": Negotiating State Authority in University Administration. Cultural Studies Assoc. Conf. (2013).

13. Cohen, S., Kamarck, T., and Mermelstein, R. A global measure of perceived stress. Journal of Health and Social Behavior 24, 4 (1983), 385-396.

14. Deutsch, M.B., Green, J., Keatley, J., Mayer, G., Hastings, J., and Hall, A.M. Electronic medical records and the transgender patient: Recommendations from the World Professional Association for Transgender 
Health EMR Working Group. $J$ of the American Medical Informatics Assoc. 20, 4 (2013), 700-703.

15. DiMicco, J.M. and Millen, D.R. Identity management: Multiple presentations of self in Facebook. Proceedings of GROUP (2007).

16. Dimond, J.P., Fiesler, C., and Bruckman, A.S. Domestic violence and information communication technologies. Interacting with Computers 23, 5 (2011), 413-421.

17. Donath, J.S. Identity and Deception in the Virtual Community. In Communities in Cyberspace. Routledge, 1998.

18. Duggan, M. and Smith, A. Social Media Update 2013. Pew Research Center's Internet \& Amer. Life Project. www.pewinternet.org/2013/12/30/social-media-update2013/.

19. Ellison, N.B., Steinfield, C., and Lampe, C. The benefits of Facebook "friends:" Social capital and college students' use of online social network sites. $J$ of Computer-Mediated Comm. 12, 4 (2007), 1143-1168.

20. Evans, M., Donelle, L., and Hume-Loveland, L. Social support and online postpartum depression discussion groups: A content analysis. Patient Education and Counseling 87, 3 (2012), 405-410.

21. Facebook Diversity. Facebook.com, 2014. www. facebook.com/photo.php?fbid=567587973337709.

22. Factor, R.J. and Rothblum, E.D. A study of transgender adults and their non-transgender siblings on demographic characteristics, social support, and experiences of violence. Journal of LGBT Health Research 3, 3 (2008), 11-30.

23. Farnham, S.D. and Churchill, E.F. Faceted identity, faceted lives: Social and technical issues with being yourself online. Proceedings of CSCW (2011).

24. Ferraro, R. Facebook introduces custom gender field to allow users to more accurately reflect who they are. GLAAD, 2014. http://www.glaad.org/blog/facebookintroduces-custom-gender-field-allow-users-moreaccurately-reflect-who-they-are.

25. George, L.K. Sociological perspectives on life transitions. Annual Review of Sociology 19, 1 (1993), 353-373.

26. Goffman, E. Presentation of Self in Everyday Life. Doubleday Anchor Books, New York, NY, 1959.

27. Grant, J.M., Mottet, L.A., Tanis, J.D., Harrison, J., Herman, J.L., and Kiesling, M. Injustice at Every Turn: A Report of the National Transgender Discrimination Survey. National Center for Transgender Equality and National Gay and Lesbian Task Force, 2011.

28. Gross, B.M. and Churchill, E.F. Addressing constraints: Multiple usernames task spillage and notions of identity. Proc. CHI Ext. Abstracts (2007).
29. Hendricks, M.L. and Testa, R.J. A conceptual framework for clinical work with transgender and gender nonconforming clients: An adaptation of the Minority Stress Model. Professional Psychology: Research and Practice 43, 5 (2012), 460-467.

30. Jankovic, C. and Haimson, O.L. Transdisciplining the Problem: Addressing Administrative Violence in University Information Systems. Cultural Studies Association Conference (2013).

31. Kannabiran, G. Themself: Critical analysis of gender in Facebook. CHI workshop paper (2011).

32. Lampinen, A., Tamminen, S., and Oulasvirta, A. All my people right here, right now: Management of group co-presence on a social networking site. Proceedings of GROUP (2009).

33. Lovibond, S.H. and Lovibond, P.F. Manual for the Depression Anxiety Stress Scales (2nd ed.). Psychology Foundation, 1995.

34. Marwick, A.E. and boyd, d.m. I tweet honestly, I tweet passionately: Twitter users, context collapse, and the imagined audience. New Media \& Society 13, 1 (2011), 114-133.

35. Marwick, A.E. I'm more than just a Friendster profile: Identity, authenticity, and power in social networking services. Association for Internet Researchers (2005).

36. Massimi, M., Dimond, J.P., and Le Dantec, C.A. Finding a new normal: The role of technology in life disruptions. Proceedings of CSCW (2012).

37. Norval, C., Arnott, J.L., Hine, N.A., and Hanson, V.L. Purposeful social media as support platform: Communication frameworks for older adults requiring care. Proceedings of PervasiveHealth (2011).

38. Sas, C. and Whittaker, S. Design for forgetting: Disposing of digital possessions after a breakup. Proceedings of CHI (2013).

39. Smith, M.E., Nguyen, D.T., Lai, C., Leshed, G., and Baumer, E.P.S. Going to college and staying connected: Communication between college freshmen and their parents. Proceedings of CSCW (2012).

40. Stutzman, F., Capra, R., and Thompson, J. Factors mediating disclosure in social network sites. Computers in Human Behavior 27, 1 (2011), 590-598.

41. Stutzman, F.D. Networked Information Behavior in Life Transition. 2011. http://gradworks.umi.com/ 34/56/3456300.html.

42. Suchman, L. Do categories have politics? Computer Supported Cooperative Work (CSCW) 2, 3 (1993), 177-190.

43. Tamir, M., John, O.P., Srivastava, S., and Gross, J.J. Implicit theories of emotion: Affective and social outcomes across a major life transition. $J$ of Personality and Social Psychology 92, 4 (2007), 731-744. 
44. Turkle, S. Cyberspace and Identity. Contemporary Sociology 28, 6 (1999), 643-648.

45. Van House, N.A. Feminist HCI meets Facebook: Performativity and social networking sites. Interacting with Computers 23, 5 (2011), 422-429.
46. Wang, Y., Leon, P.G., Acquisti, A., Cranor, L.F., Forget, A., and Sadeh, N. A field trial of privacy nudges for Facebook. Proceedings of CHI (2014).

47. Wang, Y., Norcie, G., Komanduri, S., Acquisti, A., Leon, P.G., and Cranor, L.F. "I regretted the minute I pressed share": A qualitative study of regrets on Facebook. Proceedings of SOUPS (2011).

\section{APPENDIX. ONLINE SURVEY}

1. Do you consider yourself to be transgender and/or gender non-conforming in Yes; No any way?

2. Did you have a social network site profile prior to transitioning? Yes; No

[If participant answered No to question 1 or 2, they were disqualified and exited the survey.]
3. Do you use Facebook?
Yes; No

[If participant answered No to question 3, they skipped the questions about Facebook and answered questions instead about the SNS that they used most frequently.]

4. In a typical day, about how much time do you spend using Facebook?
10 minutes or less
$10-30$ minutes
$31-60$ minutes
$1-2$ hours
$2-3$ hours
3 or more hours

5. About how many "friends" or connections do you have on Facebook? If you $0-10$ are not sure, please give your best guess.

6. In what year did you begin your gender transition on Facebook? Although it [List of years 2004 - 2014] may be difficult to pinpoint a particular year, please estimate the year in which you first began to change your name, gender, and/or profile picture appearance on Facebook.

7. Which of the following have you done in order to transition on Facebook? [Multiple options possible]
Announced transition (e.g., a status update) Changed the gender listed on my profile Changed the name displayed on my profile Changed my profile picture Deleted my profile or account Created a new profile

Other (please specify)

8. Which of the following have you done with your friends list on Facebook during your gender transition?

[Multiple options possible]
"Unfriended" or blocked people who may not be supportive

"Unfriended" or blocked people who I didn't want to know about my transition

Made some posts visible only to some people in my network

Created groups or "circles" to make posts visible to only certain groups of people (e.g. to share gender transition information with only those people)

Other (please specify) 
9. How stressful was it to transition in each of these environments? 1-Not at all stressful

a. Facebook

2-Slightly stressful

3-Moderately stressful

4-Extremely stressful

N/A

[Other environments included in survey but not relevant for this paper]

10. Roughly how many people in your Facebook network were supportive of your 1-None transition online?
2-Almost none
3-Few
4-About half
5-Most
6-Almost all
7-All

11. What parts of transitioning on Facebook were especially difficult? Why were [Open-ended response] they difficult?

12. What do you think would improve the online gender transition experience on [Open-ended response] Facebook?

13. What would you change about Facebook to make it more friendly to [Open-ended response]
[O transgender / gender non-conforming people?

14. Is there anything else you would like to tell us about your transition [Open-ended response]
[ experience on Facebook?

15. Did you change your gender to a Custom gender option on Facebook? Yes; No

16. Did you try to enter a Custom gender option that was not recognized by Yes; No Facebook?

17. To what extent do you agree with the following statements:

a. Facebook's Custom gender options improve the online gender transition 2-Disagree experience.

1-Strongly Disagree

3-Undecided

4-Agree

5-Strongly Agree

[Other statements included in survey but not relevant for this paper]

18. Is there anything else you would like to tell us about your thoughts about and [Open-ended response] experience with Facebook's Custom gender options and/or Neutral pronoun options?

19. How stressful was it to transition in each of these environments?

a. Among close friends

b. Among all friends

c. Among close family (e.g. parents, children)

d. Among extended family (e.g. grandparents, aunts, uncles, cousins, nieces, nephews, etc.)

e. At work

f. At school

20. Is there anything else you would like to tell us about any stress that you may [Open-ended response] have experienced as part of your transition offline and/or online?

21. Roughly how many of your friends were supportive of your transition offline 1-None

$1-$ Not at all stressful

2-Slightly stressful

3-Moderately stressful

4-Extremely stressful

N/A (e.g. in- person, on the phone, etc.)?

2-Almost none

3-Few

4-About half

5-Most

6-Almost all

7-All

22. Roughly how many of your family members were supportive of your transition offline (e.g. in-person, on the phone, etc.)?
1-None

2-Almost none 
3-Few

4-About half

5-Most

6-Almost all

7-All

23. Roughly how many of your co-workers were supportive of your transition offline (e.g. in-person, on the phone, etc.)?

1-None

2-Almost none

3-Few

4-About half

5-Most

6-Almost all

7-All

N/A

24. Is there anything else you would like to tell us about the support that you received during your transition?

[Open-ended response]

25. What is your current gender identity?

[Multiple options possible]

Male

Female

Transmale/transman/FTM

Transfemale/transwoman/MTF

Genderqueer/gender-non-conforming

Different identity (please state)

26. What is your sex assigned at birth?

Male

Female

Other

\section{With which racial or ethnic group(s) do you most identify?}

[Multiple options possible]

American Indian/Native American

Asian/Pacific Islander

Black/African American

Hispanic/Latino

White/Caucasian

28. In what year were you born?

[List of years]

29. In what year did you begin your gender transition? Although it may be difficult to pinpoint a particular year, please estimate the year in which you first began to change your physical appearance, name, and/or pronoun.

30. What is the highest degree or level of school you have completed?

[List of years]

No schooling completed

Elementary school to 8 th grade

Some high school, but did not finish

High school graduate, diploma or the equivalent (for example: GED)

Some college, but did not finish

Trade/technical/vocational training

Associate degree

Bachelor's degree

Master's degree or professional degree

Doctorate degree

31. What is your annual household income, before taxes?
Under $\$ 10,000$
$\$ 10,000-\$ 19,999$
$\$ 20,000-\$ 29,999$
$\$ 30,000-\$ 39,999$
$\$ 40,000-\$ 49,999$
$\$ 50,000-\$ 74,999$
$\$ 75,000-\$ 99,999$
$\$ 100,000-\$ 124,999$
$\$ 125,000-\$ 149,999$
Over $\$ 150,000$
Prefer not to disclose
Not sure 